Usage, Usability, and Utility of 3D City Models, 02001 (2012)

DOI: $10.1051 / 3 \mathrm{u} 3 \mathrm{~d} / 201202001$

(c) Owned by the authors, published by EDP Sciences, 2012

\title{
Measuring the impact of 3D data geometric modeling on spatial analysis: Illustration with Skyview factor
}

\author{
M. Brasebin ${ }^{1, a}$, J. Perret ${ }^{1, b}$, S. Mustière ${ }^{1, c}$ and C. Weber ${ }^{2, d}$ \\ ${ }^{1}$ IGN, COGIT, 73 avenue de Paris, Université Paris-EST, 94165 Saint Mande Cedex, France \\ ${ }^{2}$ Laboratoire Image, Ville, Environnement - ERL 7230, 3 rue de l'Argonne, \\ 67083 Strasbourg, France
}

\begin{abstract}
The increased availability of 3D urban data reflects a growing interest in 3D spatial analysis. As 3D spatial analysis often uses complex 3D data, studies of the potential gains of using more detailed 3D urban databases for specific uses is an important issue. First, more complex data implies an increase in time and memory usage for the analysis (and calls for more research on the efficiency of the algorithms used). Second, detailed 3D urban data are complex to produce, expensive and it is important to be well informed in order to decide whether of not to invest in such data. Currently, many studies have been led about the fitness for use of $2 \mathrm{D}$ data but they are very scarce concerning 3D data. This article presents a method to determine the influence of 3D modeling on the results of 3D analysis by isolating the potential sources of errors (such as roof modeling and geometric accuracy). This method is applied on two 3D datasets (LOD1 and LOD2) and a 3D indicator (the sky view factor or SVF). The results show that the significant influence of roof modeling is globally compensated by the difference in geometric modeling but that important local variations are noticed. Nevertheless, for $75 \%$ of the SVF processed the difference between the results using these two databases is lower than $2 \%$.
\end{abstract}

\section{INTRODUCTION}

These last decades, a dramatic increase in the number of available 3D urban databases is noticed. Most of these databases are heterogeneous due to different production processes, Levels Of Detail (LOD) or specifications. Whatever the type of database, its main use is generally to provide a visual support to communicate, discuss and exchange about urban planning projects. As they are designed for such use, such databases are quite difficult to manipulate for spatial analysis: geometries usually do not meet the prerequisites to allow the direct application of geometric operators; they contain very few attributes, etc. For example, a few hidden faces may be missing and normal vectors may have their direction inversed. Thus, due to these difficulties, analysis tools usually require a certain level of expertise on the validation and the repair of 3D urban data. However, these 3D databases could convey new information to better understand and study the city through specific indicators linked to urban morphology for instance. Such knowledge can then potentially be used for the support of decision processes and have a significant impact on the future of our cities.

Many 3D indicators were elaborated from observations of the real world (see $[1,2]$ for urban environmental morphologic indicators or [3] for the characterization of building-landmark). The

\footnotetext{
${ }^{a}$ e-mail: mickael.brasebin@ign.fr

be-mail: julien.perret@gmail.com

ce-mail: sebastien.mustiere@ign.fr

de-mail: christiane.weber@live-cnrs.unistra.fr
}

This is an Open Access article distributed under the terms of the Creative Commons Attribution License 2.0, which permits unrestricted use, distribution, and reproduction in any medium, provided the original work is properly cited. 
development of 3D data and GIS is a great opportunity to apply them at the scale of an urban block, a district or an urban area. Nevertheless, it is important to assess the potential error of an indicator due to data capture approximations, modeling choices or specification choices. Once such errors are known, their influence on the results of an analysis used in a decision process can be taken into account. On the one hand, knowing the influence of the quality of the data used to compute an indicator can be used to apprehend its impact on the results and weight the results accordingly. On the other hand, given a certain requirement on the precision of the results for a certain study, such knowledge can be used to chose the most appropriate 3D database and the corresponding production process. From a pragmatic point of view, as LOD1 (according to [4]) databases, i.e. extruded building footprints for the geometries of buildings, are a lot cheaper than more detailed ones (with roof structures, architectural details, etc.), the issue is also to know if the gain of quality is significant enough to invest money in high quality data for a given study. Furthermore, it is interesting to know if the additional computation time and memory usage are worthwhile.

The aim of this article is to present a method that assesses the impact of 3D data geometric modeling on indicator computations applied to a given indicator: the sky view factor (SVF) [5]. To begin with, a brief state of art will present the different approaches used to evaluate $3 \mathrm{D}$ data quality as well as our methodology and original proposal (section 2). Then, the chosen indicator and the 3D urban test data used in our study are introduced in section 3. An illustration of our method on this indicator is presented before discussing our results and finally suggesting perspectives (sections 4 and 6 respectively).

\section{3D DATA QUALITY}

\subsection{State of art}

Two main types of data quality can be identified according to [6]: internal quality (satisfaction of data specifications) and external quality (adaptation of data for a given use). Concerning internal quality, elements generally considered are those defined by ISO specifications [7]: "geometric accuracy, attribute accuracy, completeness, logical consistency, semantic accuracy, lineage and temporal accuracy". In the field of 3D data research, many studies have been led in order to detect and correct geometric errors. [8] proposes algorithms to automatically clean the 3D topology of geometric primitives, [9] conceptualize this issue to validate geometry in an Oracle database and [10] studied the different geometric errors to finally classify them into 10 categories. In $[11,12]$, an architecture is proposed to check the topological quality of data. It provides some report of quality about geometric errors of a 3D model and a report about the operations required to correct them. A majority of work about internal quality concerns the producer and not the user.

Concerning 3D data external quality, [13] notice that the difference of total annual solar irradiation assessed in a 3D model differs of only $1 \%$ between LOD1 and LOD2 models. Nevertheless, the difference reaches $6 \%$ for the summer solstice and $-33 \%$ for the winter solstice. [14] studies the influence of a modeling by DEM or by LOD1 buildings on Sky View Factor values. He notices an error of 0.02 on $15 \%$ of the studied data. [15] studies the difference between Sky View Factor values computed using a 3D database to real world values. He observes some differences but considers the results of the values computed with the 3D database close enough to the ground truth for his needs. [16] compare the trajectory of landslides modeled in 2D5 or 3D and notice some differences up to $12 \%$ about the landslide lengths. [17] proposes an algorithm for reconstructing buildings using LIDAR and 2D data. The authors also study the impact of the reconstruction algorithm parameters on several morphological indicators, such as building volumes, facade areas and roof areas. Due to the reconstruction process, the buildings produced by the proposed algorithm always have similar geometric properties and do not show the wide variety of modeling choices that one can find when using several 3D data sources.

In the context of 2D data external quality, several studies have been carried in order to estimate the precision of specific computations: for instance, [18] proposes a statistical model to asses the error of 
polygon area calculation and [19] introduces the estimation of digitization error on distance and area value (see [20] for a complete state of art on fitness for use on 2D datasets). Finally, [21, 22] proposes to estimate the impact of the different source of geometric errors (i.e. polygonal approximation, 2D5 or 2D modeling) on length calculations for 2D linear data (such as roads). His method consists in isolating the different sources of error in geometry modeling to asses their respective influences. Nevertheless, to the best of our knowledge, no study has yet focused on the influence of the different sources of errors in $3 \mathrm{D}$ data as it exists for 2D data.

\subsection{Proposal and method}

Currently, many studies offer relevant knowledge about the impact of 2D geometric modeling on measure computations, but works on 3D data, focusing on external quality, are scarce. However, the large variety of 3D city object modeling types offers many dimensions to explore in order to understand the influence of the variation of the outputs. We propose in this article to apply methods of 2D external quality analysis on 3D geometries. In the next section, we present our method to study these influences. An illustration of the method application is presented in section 4 on the SVF. The interest of this indicator is to only consider geometry (i.e. no attribute is used) and that its variations cannot be directly inferred from data specifications.

\section{PROPOSED METHOD}

The aim of this article is to present a generic method to test the influence of the different contributions of geometric modeling for values of $3 \mathrm{D}$ indicators. Our idea considers indicator calculations from a reference database and a database to be assessed. In order to determine the influence of geometric errors, comparison of indicator values is processed in the two input databases in order to detect the different sources of geometric errors. Then, intermediate databases that attempt to isolate different sources of error are produced in order to compare their indicator values to the values obtained using the reference database.

Our proposed method can be decomposed into 5 steps:

1. Calibration of the algorithm (section 3.1),

2. Comparison of indicator values from the input databases,

3. Determination of potential sources of difference (section 3.2),

4. Production of intermediate databases isolating different sources of error (section 3.3),

5. Comparison of indicator values from intermediate databases with the values from the reference database.

We can notice that steps from 3 to 5 can be iterated: if, during step 5, new sources of errors (undetected in step 2) appear, the process goes back to step 3. The comparison method is adaptable according to the indicator and can integrate specific indicator parameter inputs.

\subsection{Uncertainty sources in the algorithm input}

Our method is based on the evaluation of the impact of the data on a specific indicator. Nevertheless, we first have to make sure that the indicator is not biased by its own parameters. Such a process is usually referred to as sensitivity analysis. In fact, many implementations of algorithms for 3D indicator calculation include heuristics or simplifications in order to make the evaluation of the indicator faster of simply computable. Such parameters can, for instance, reduce the search space, the data taken into account, the number of samples used for an estimation, etc.

Figure 1 illustrates a generic algorithm and its inputs: a dataset and input parameters. The first step of out method consists in defining the parameter space for the algorithm implementing the indicator being used and computing the values of the indicator on a wide range of values using the reference 


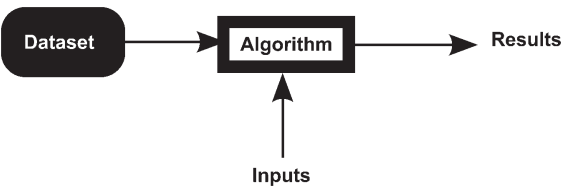

Figure 1. Schema of an algorithm.

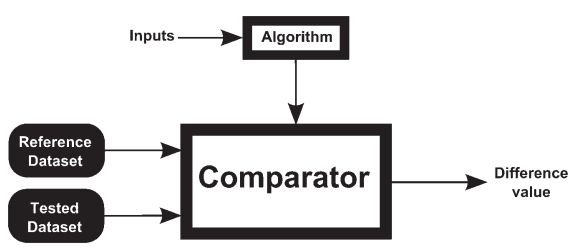

Figure 2. Algorithm comparison.

dataset. This step helps us to identify the parameters that will be used in the following steps and their influence on the results.

\subsection{Uncertainty sources in the data}

The second step involves the computation of the indicator on the input datasets and the comparison of the results (figure 2). This step, along with prior knowledge of the databases and of the indicator, helps us in identifying possible sources of uncertainty in the data (third step). This is a key step of the method. For instance, for the SVF, possible sources of differences between databases are: planimetric precision, altimetric precision, roof modeling, building specifications (individual buildings or aggregated ones, etc.) and building capture specification (e.g. which buildings are captured and which are ignored).

\subsection{Isolation of uncertainty sources}

Using the results of step 3, we propose to produce intermediate databases (step 4) in order to better isolate the sources of uncertainty in the modeling of the data. For instance, we can produce databases that attempt to remove the uncertainty due to roofs, building selection and planimetric accuracy. Once these databases are produced, the indicator values are computed and their values compared to the reference values (step 5, illustrated in figure 3).

\section{RESULTS AND DISCUSSION}

In this section, we apply the proposed approach on a test area, using two 3D building datasets and the SVF as the indicator. Section 4.1 deals with the prerequisites of the application of the method and section 4.2 presents the Sky View Factor indicator. Section 4.3 presents step 1 of our method and step 2 to 5 are described in section 4.4. A global (section 4.5) and a local (section 4.6) analysis of these results offer an understanding of the method output. Section 5 presents another study considering SVF from points on building facades.

\subsection{Preparing the databases}

The test area used in this article in located in the center of the city of Strasbourg in France. 


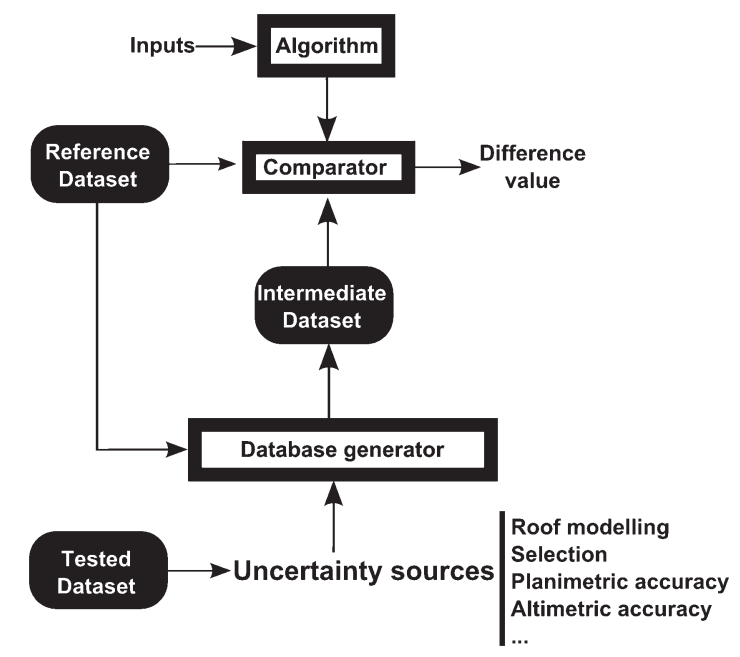

Figure 3. Isolation of uncertainty sources.

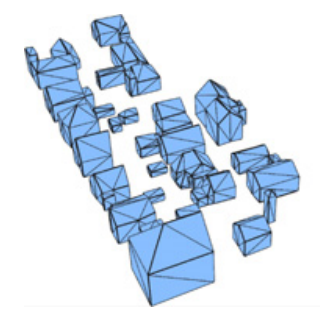

(a) Non-textured 3DDB illustration

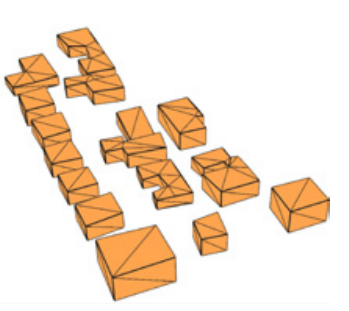

(b) BDTOPO illustration

Figure 4. The two databases in the same extent.

The two datasets used (figure 4) are: the 3D database produced by the Strasbourg agglomeration, which is composed of 3D buildings with roofs, roughly corresponding to CityGML LOD2 (we will henceforth refer to it as 3DDB) and the BDTOPO® database, that covers the whole French territory, produced by the French National Institute of Geographic and Forestry Information (IGN France), which is composed of 3D roof edges extruded using a DTM, corresponding to CityGML LOD1.

The indicator used is the SVF, presented in section 4.2. This indicator has to be evaluated on a sample of points. Therefore, we generate points on the dataset using a 40 meter grid and selecting the points which are not inside the buildings. This set of points is then enriched by the addition of points located on the road network with a step of 30 meters (in order to have some points in the high building density zones). The altitude of the points initially on the DTM (provided with each database) is raised by $2.5 \mathrm{~m}$ in order to stress the potential influence of roof modeling. The generated points are illustrated in figure 5. Finally, minor actuality differences between the datasets were corrected by hand. The automation of such a process or the integration of its impact (sensitivity analysis) will be the object of future work.

\subsection{Sky View Factor}

The Sky View Factor (or SVF) is an indicator mentioned in [5] in the context of the evaluation of the urban heat island effect. The SVF corresponds to the proportion of visible sky from a given ground point 


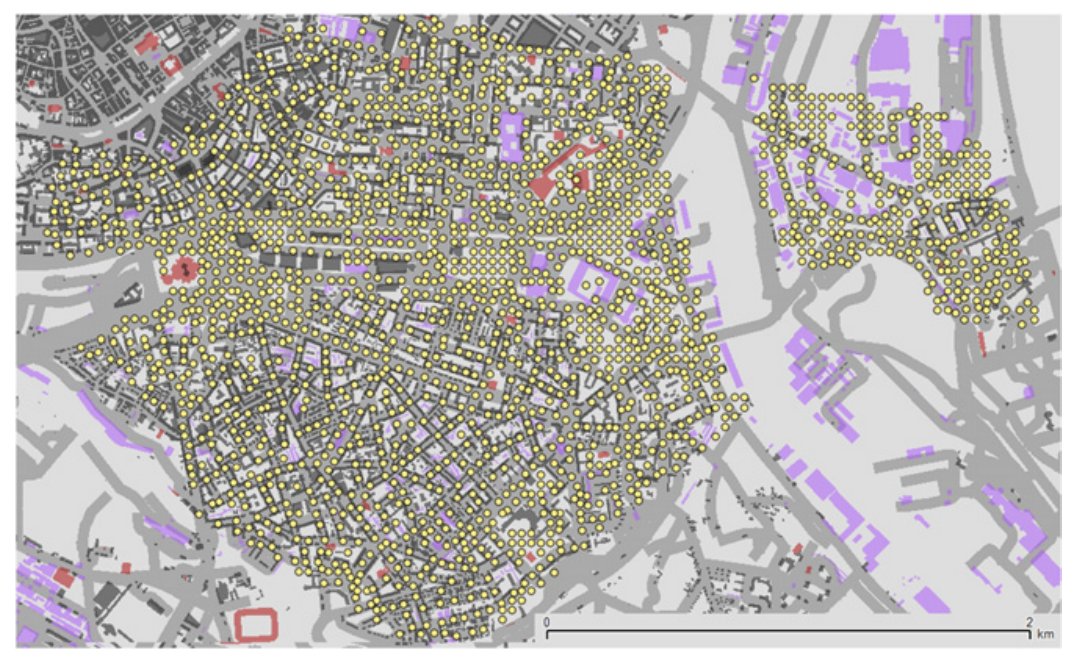

Figure 5. Situation map of the zone.

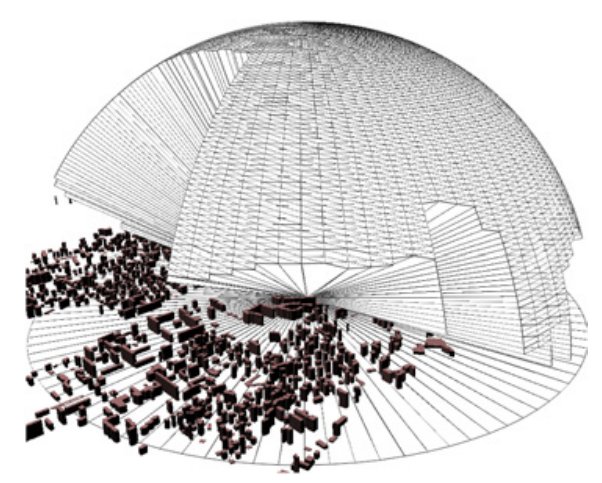

Figure 6. Sky View Factor is the ratio between the area of open-space and the total area of the hemisphere.

in the upper hemisphere (figure 6). Johnson also proposes an analytic method to determine this factor. Different methods are used to determine the value of this indicator: (i) real city models, (ii) analytic methods, [23] notably simplify it by a ratio between the height and the width of empty space from a viewpoint, (iii) fish-eye pictures [15], (iv) spherical projection [24], (v) ray casting [25].

The SVF has several uses. In climatology, a relationship between the urban heat island effect higher temperature in cities than in nature) has been shown [26, 27] and is used in environmental tools [28]. In architecture, [29] uses the SVF to study the visual impact of urban shapes and sky shapes to characterize the empty space in a regulation context. [30] continues this work in order to study the variation of city ambiance along a pedestrian trajectory. [31] uses the SVF and sky shape analysis to reinterpret Lynch's [32] visual elements.

\subsection{Determination of algorithm parameters}

The first step is to determine the parameters of the SVF algorithm. Our method is processed by raycasting as we consider that this method is relevant as a basic functionality included in different 3D GIS implementations. In our experiments, GeOxygene 3D module [33] is used for our implementation. 2 parameters are considered: the angular step for which ray castings are processed and the radius where 


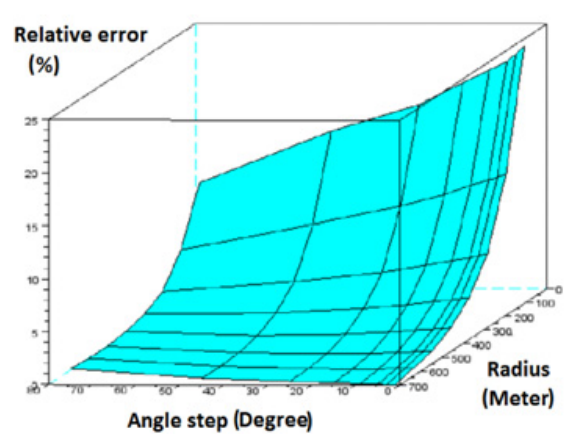

(a) BDTOPO

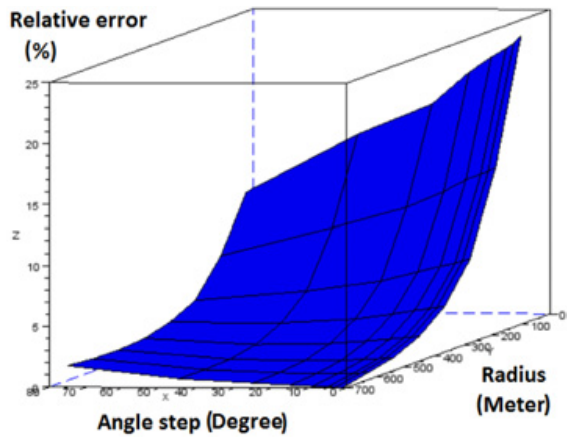

(b) $3 \mathrm{DDB}$

Figure 7. Relative error in $\%$ of SVF according to angle step $\left(^{\circ}\right)$ and radius considered (m).

buildings are considered. For each database, a reference value is evaluated on a set of points with the most demanding (i.e. with less approximation) parameters (50 points in point set, $700 \mathrm{~m}$ radius and $0.4^{\circ}$ in our study) in terms of computation time. Then, different SVF calculations are processed considering rougher parameter values on the set of points and compared to reference values. Figure 7 presents the average of relative errors to reference points on our two databases. We can notice that the influence of the radius is the most important. We chose for our experiment the couple of value $600 \mathrm{~m}$ and $5^{\circ}$ as it corresponds to values with low average error ( $0.4 \%$ in 3DDB and $0.38 \%$ in BDTOPO), because none of the tested points has an error higher than $1 \%$ and finally that these parameters provide us with good performances (decrease in the number of ray castings compared to using $0.4^{\circ}$ ).

\subsection{Differences calculation and intermediate databases generation}

Figure 8 shows the differences in SVF values for the sample points on the two input datasets: the 3DDB and the BDTOPO datasets. On this figure, we can clearly see that the SVF in very open places of the city are very close from one dataset to the other. This result is not surprising considering that, in such places, the quality of the building representations little impacts the results of the SVF. We can also see clusters of points where the SVF is higher in the 3DDB than in the BDTOPO or the other way around.

Figure 9 illustrates the different configurations and the sources of these indicator differences. Aside from building selection differences due to different specifications, the modeling of the roofs seems to explain most of the cases where the SVF is lower in the 3DDB. Concerning planimetric precision, the buildings of the BDTOPO tend to be larger than 3DDB ones, thus narrowing streets and impacting the SVF values. This is actually not only a question of precision, but also a specification difference: when the 3DDB uses the building footprints from cadastral map, the BDTOPO actually defines roof edges as the building geometry, thus widening the buildings.

Using the identified sources of uncertainty, two intermediate datasets were produced (figure 10): the first, IDB1, by removing buildings from the 3DDB where building selection was identified in the BDTOPO (usually small buildings with an area below the specifications of the database), the second, IDB2 by further removing the roofs.

Finally, the indicator values and their differences were computed on all datasets.

\subsection{Differences calculation between intermediate databases: Global analysis}

The different results are presented in figure 11. The differences of SVF in 3DDB and IDB1 (figure 11a) is globally low on the zone except on some particular zones. As the difference between these two 


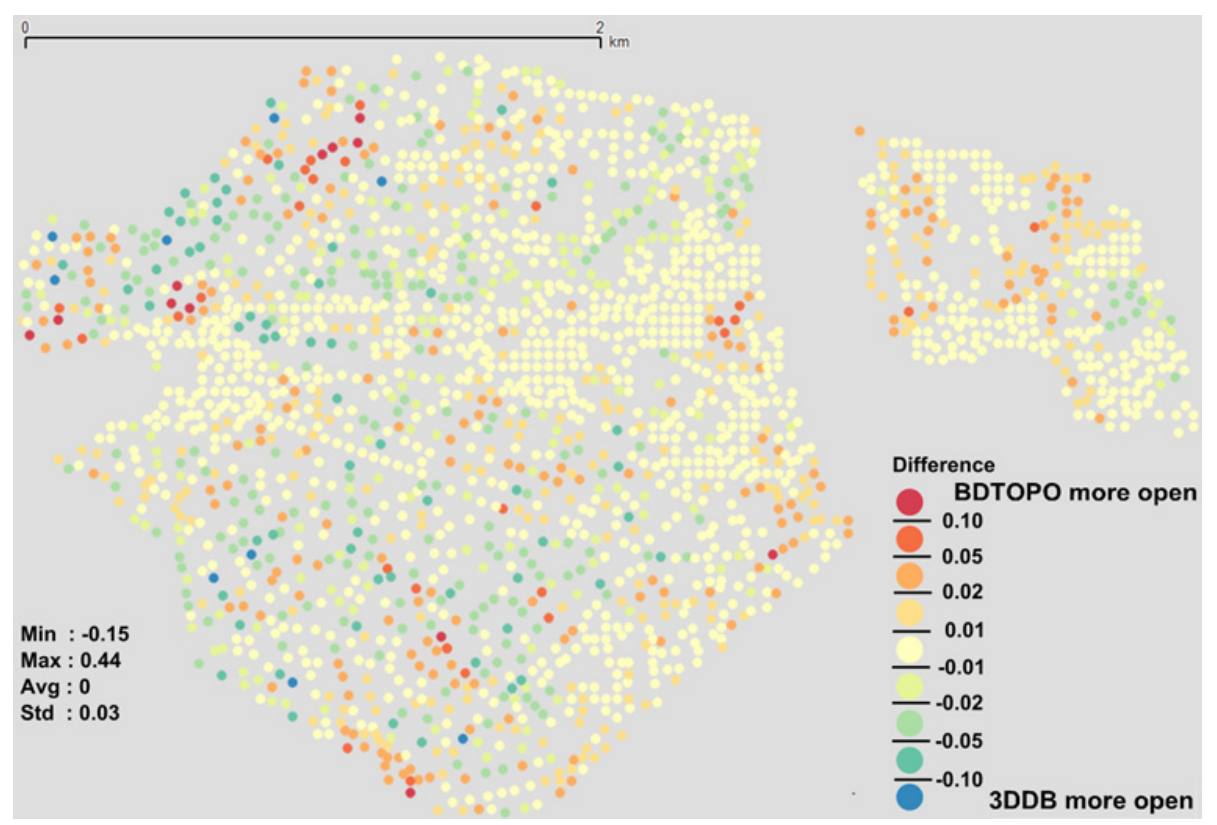

Figure 8. Difference of SVF on our two databases. Red colors correspond to SVF higher in BDTOPO and blue colors to SVF higher in 3DDB.

databases was the presence of small buildings, this result can be explained by the fact that if these buildings are removed and as this zone has a high density, an other building behind the small will offset the absence of the small building. Points with high differences are explained by the proximity of small buildings. The differences between IDDB1 and IDDB2 (figure 11b) are globally more significant. The average of differences is about 0.03 (that means that the sky is $3 \%$ more visible in IDB2 than in IDB1) with some extreme values (up top 40\%). The map confirms that the influence of roof modeling is more important in high building density areas. The last map presents the difference between IDB2 and BDTOPO (figure 11c). The results show that the difference concerning geometric accuracy is quite important: $3 \%$ of the sky is visible in IDB2 and not in BDTOPO. The map shows that the influence is more important in high density zone with narrower streets in BDTOPO than in IDB2 explained by rougher and larger geometries in BDTOPO. Nevertheless, some exception are observed and presented in the next section. We notice that the average difference concerning the influence due to roof modeling is offset by the influence due to geometry modeling explaining the average difference is near to 0 of the initial difference calculation (figure 8).

As roof modeling and geometric accuracy influence all results, we will focus on them; the next results do not consider this very local error source. The repartition of differences considering classification according to error sources is shown in figure 12 for the tested points. Many points (1572) have nearly the same SVF ( $\pm 2 \%$ of difference of visible sky) in BDTOPO and 3DDB. But, for many of these points (487), this result is obtained with the combination of two significant influences: a negative influence of accuracy difference and a positive influence of roof modeling. When the sky is more visible in BDTOPO (SVF differences > 0.2), there is no major trend to explain the results with the different error sources. When the sky is more visible in 3DDB (SVF differences $<-0.2$ ), $80 \%$ of the cases are due to the impact of roof modeling and geometric accuracy. A local study is necessary to understand the spatial configuration of the errors, in the next section, we focus on local zones to explain these different configurations. 


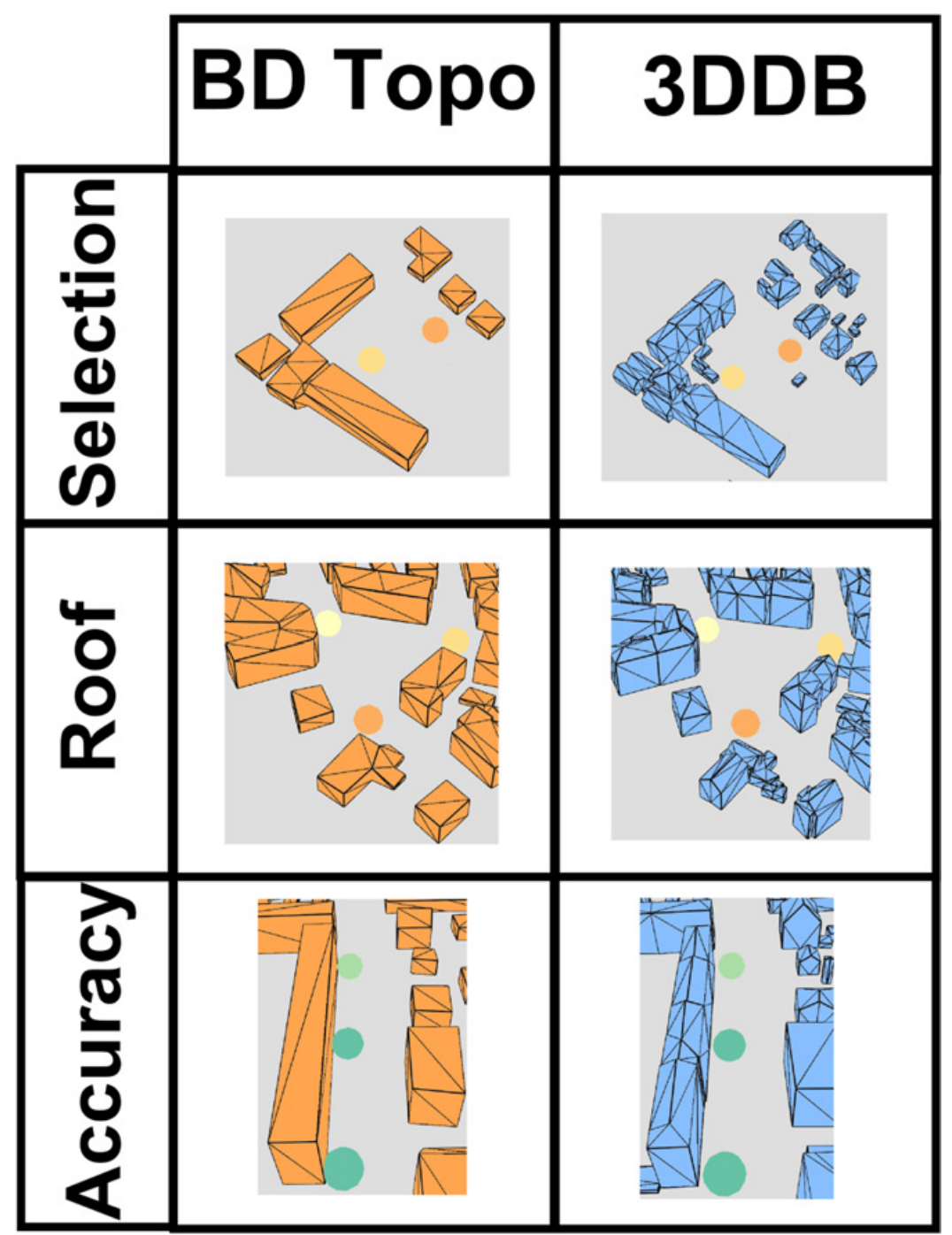

Figure 9. Different sources of influence of SVF values.

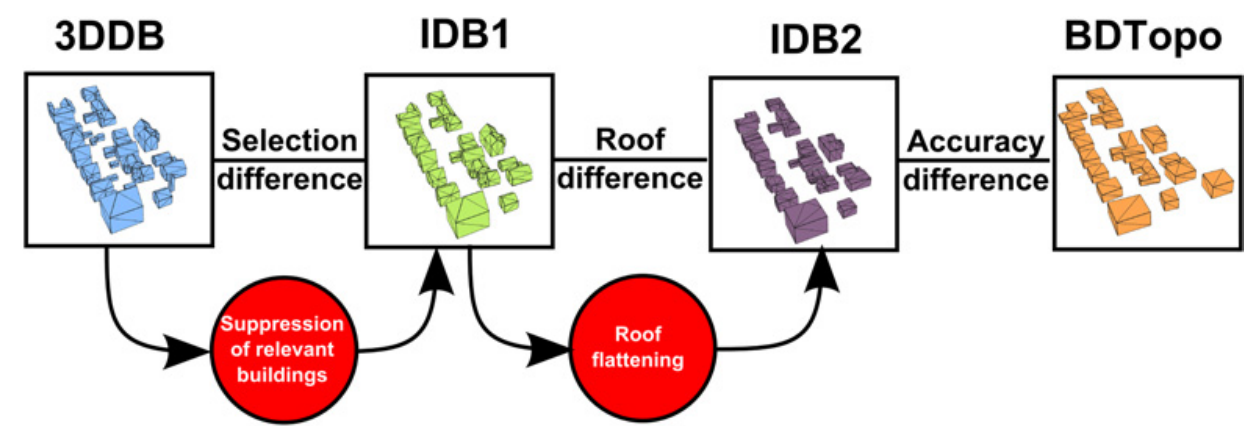

Figure 10. Production of the intermediate databases. 
Usage, Usability, and Utility of 3D City Models

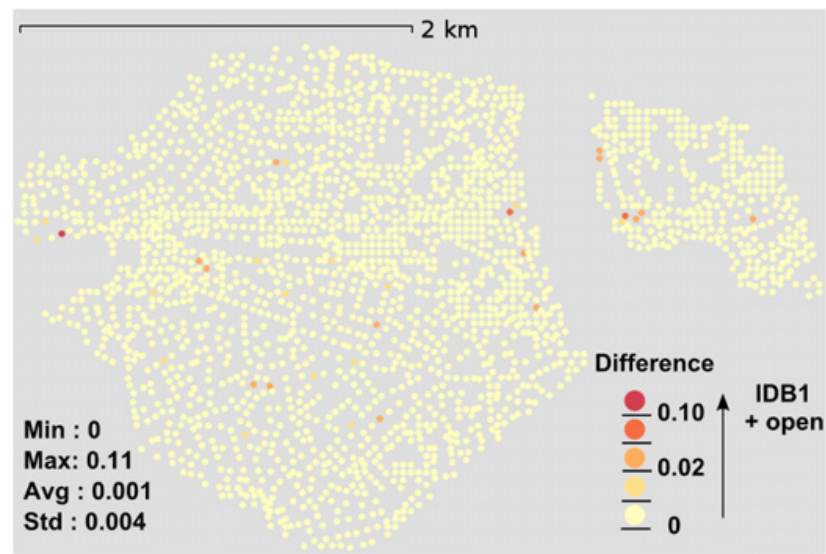

(a) Difference of SVF on 3DDB and IDB1.

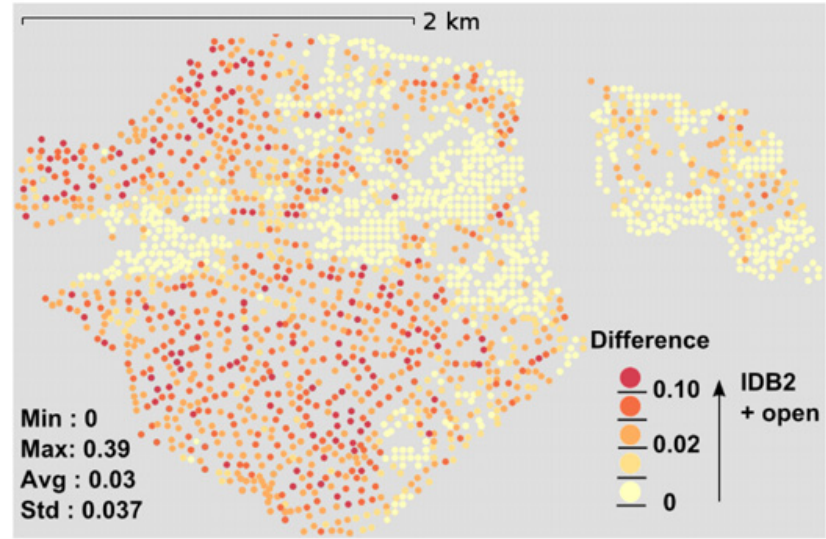

(b) Difference of SVF on IDB1 and IDB2.

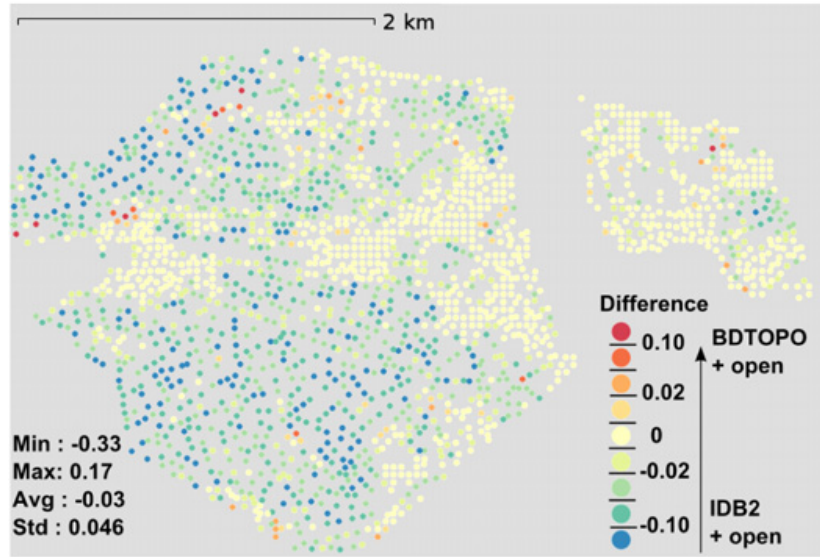

(c) Difference of SVF on IDB2 and BDTOPO.

Figure 11. SVF differences on the different datasets. 


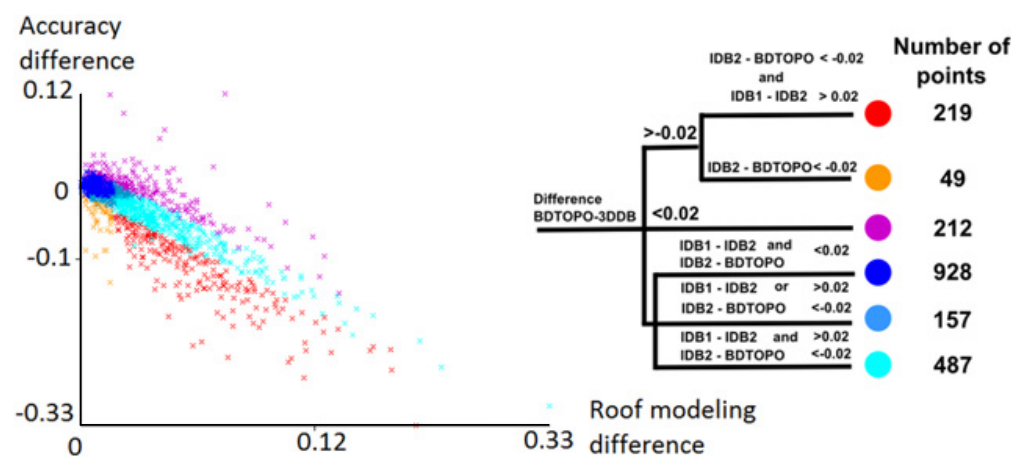

Figure 12. Classification of the different types of error considering geometric accuracy roof modeling and results between BDTopo and 3DDB.

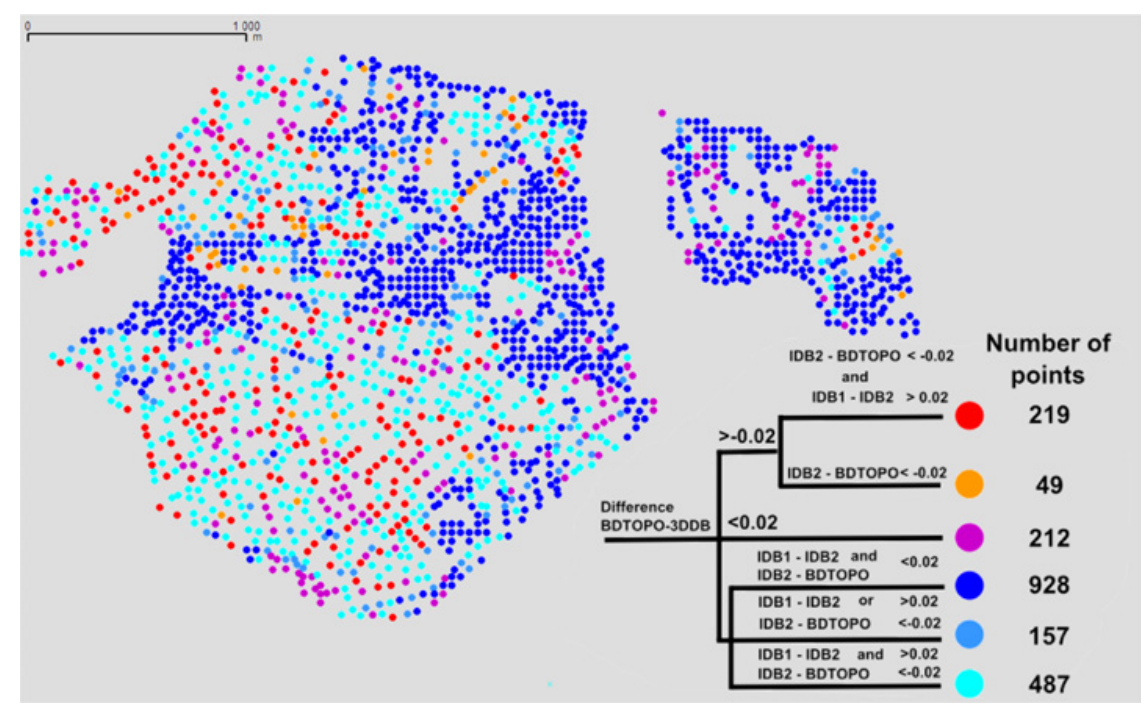

Figure 13. Repartition of the classified points in the test zone.

\subsection{Local analysis}

A map of point locations according to their classes (figure 13) shows that points with few errors and little influence of the different sources of error are located in low density areas whereas points with important influence of different sources of error are located in dense areas. Some clusters, or spots of same class points, are observed. This is due to the fact that the errors are generally the result of a building or a group of buildings influencing its nearest points. This trend is more evident for points with high positive or negative errors.

Figure 14 shows local configurations of detected classes and helps to understand the impact of the different combinations of the different sources of influence. These situations confirm some hypothesis detected during the global analysis:

- the offset of accuracy error by roof modeling (light blue class),

- important negative errors are located in areas with important influence or roof and geometric accuracy (red class),

- less important negative errors are explained notably by geometric accuracy (orange class). 
Usage, Usability, and Utility of 3D City Models

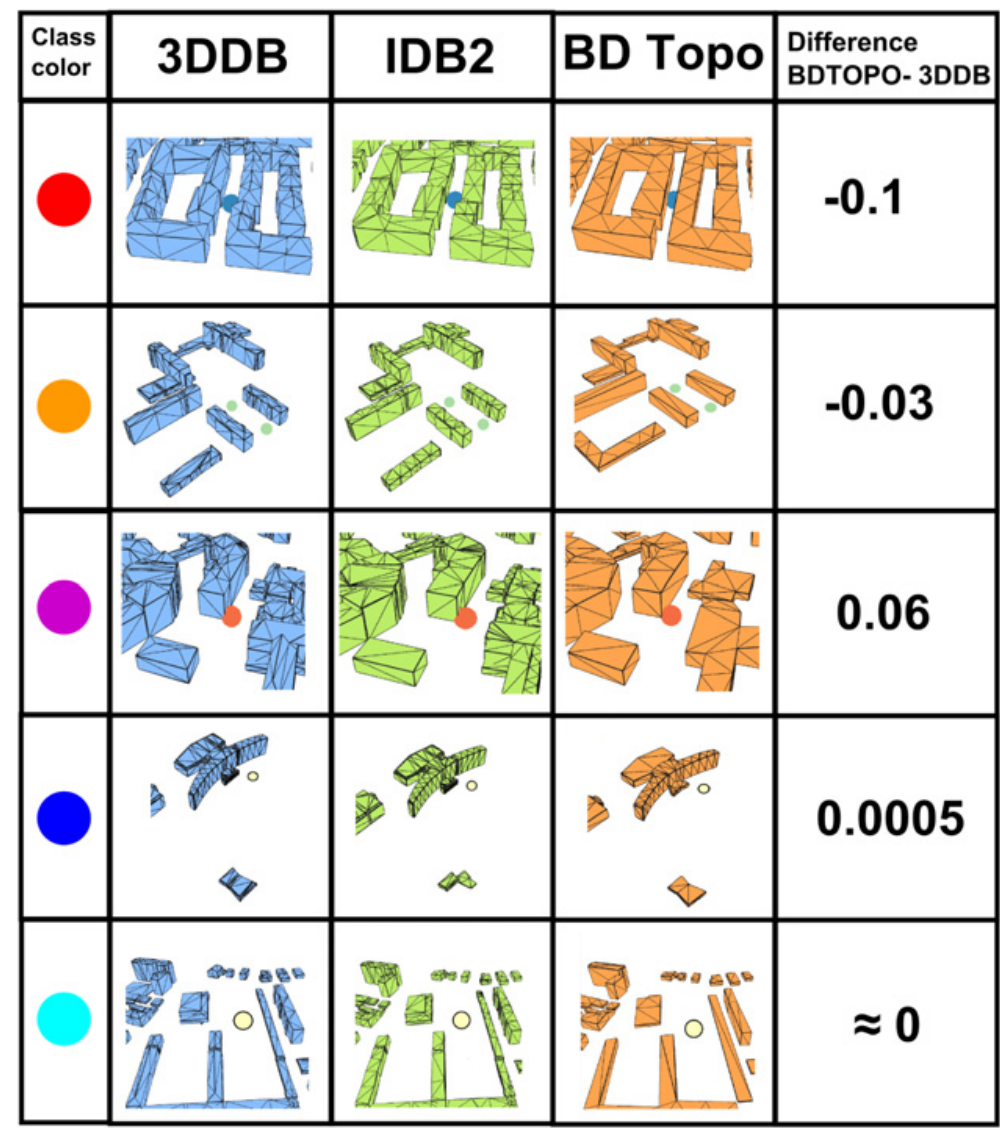

Figure 14. Different local configurations of the different classes with three databases. A color class corresponds to the keys in figure 12 and colors of points in 3D scenes to legends in figure 8.

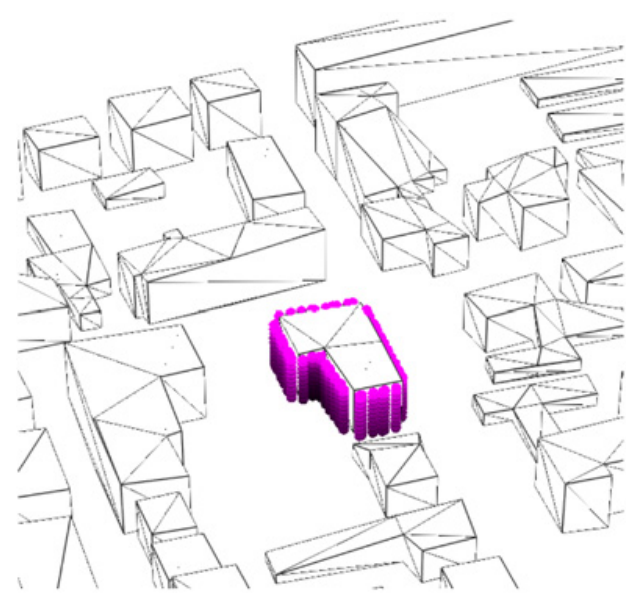

(a) BDTopo studied building

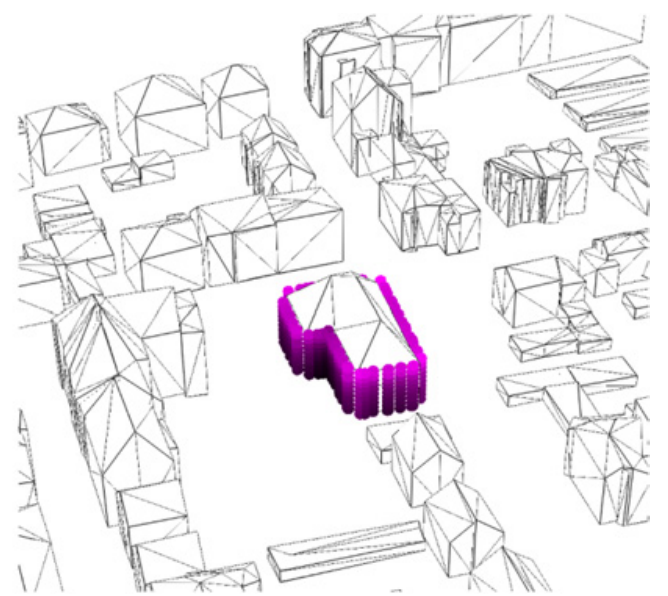

(b) Equivalent 3DDB studied building

Figure 15. Points generated on concerned building. Color value is proportional to openness. 


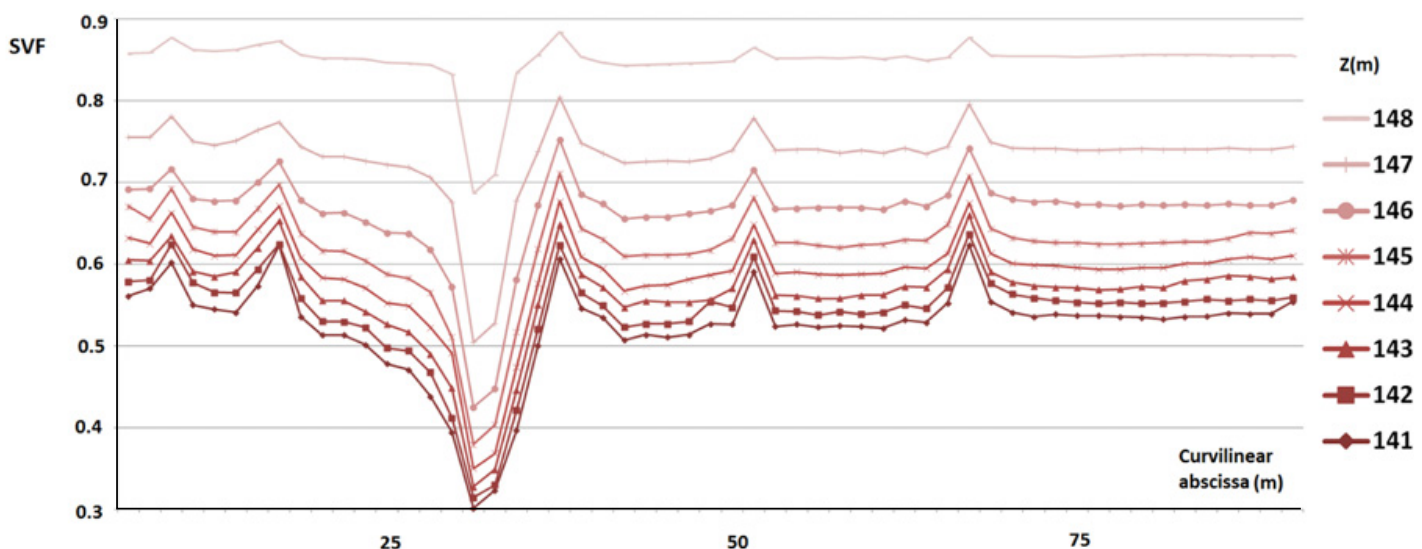

(a)

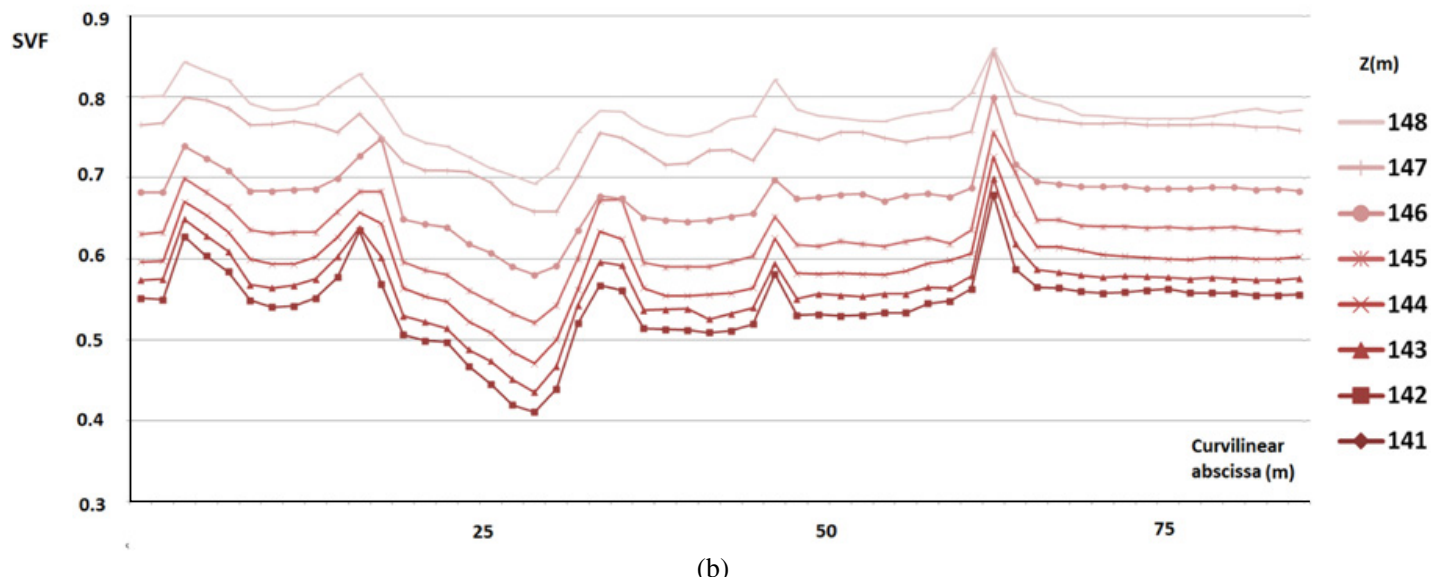

Figure 16. Sky view factor according to curvilinear abscissa and to altitude point for (a) BD Topo and (b) 3DDB.

\section{FURTHER DISCUSSION: VARIATION OF SVF WITH POINTS ON BUILDING WALLS}

The SVF could also be used in order to estimate the energy production/saving potential with respect to power generation and artificial lighting. In this context, we study the influence of modeling on the computed SVF of a point set generated on building facades. This set is generated from the intersection between a 3D grid with a $1 \mathrm{~m}$ resolution and the walls of a given building and then shifted of $0.1 \mathrm{~m}$ in the direction of the building normals. Selected building and point set are shown in figure 15. Figure 16 shows the variation of the SVF according to altitude and curvilinear coordinate along the exterior wall of the building (hereafter called curvilinear abscissa) for both databases. These graphics show that the SVF increases with altitude and the presence of six noticeable extrema. They correspond to points near the building corners that cause important variations of the SVF. The minimum is due to a reflex corner: in this configuration, the selected building hide an important part of the sky whereas the effect is the opposite for non-reflex corners. Globally, graphics are getting smoother with increasing altitude showing that the SVF tends to 1 and that local variations are flattening when the sky is more visible. The difference of SVF according to altitude at a given point can be assessed by measuring the distance between two consecutive curves. The difference between two consecutive curves constantly increases 
Usage, Usability, and Utility of 3D City Models

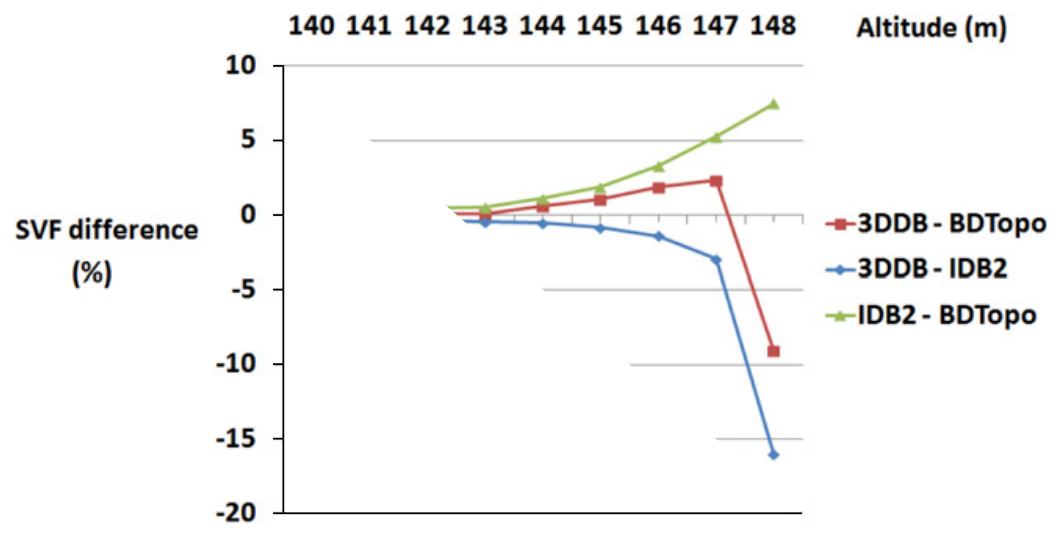

Figure 17. Difference of average SVF in \% according to altitude between 3DDB and BDTopo (in red) and between 3DDB and IDB2 (in blue).

with altitude for the BD Topo (figure 16a) whereas this phenomenon is less visible for the 3DDB (figure $16 \mathrm{~b})$. The absence of roof modeling in the BD Topo causes more important variations near the top of the wall.

By processing differences between 3DDB, IDB2 and BDTopo, the importance of the influence of the different sources can be assessed. Figure 17 illustrates that SVF is nearly the same for the different databases at the ground level but is very different near the building ridge. The average SVF difference between 3DDB and BDTopo reaches $-9 \%$ for this altitude. We can notice that the SVF difference increases until $2 \%$ for intermediate altitudes. We notice that the influence due to geometric accuracy is prevalent for lowest altitudes whereas the influence of roof modeling is higher near the building ridge (until 16\%).

\section{CONCLUSION}

In this paper, we propose a method for the evaluation of the impact of 3D geometric modeling on the computation of indicator values. Our method was implemented and tested on a test area using two input databases.

It allowed us to quantify the impact of the different sources of uncertainty in the $3 \mathrm{D}$ geometric modeling of the data. We also believe that it provided us with new insights on the specifications of 3D databases and on how such a method could help improve the design of database specifications.

The results show that the average difference of SVF values is low between the two databases. This study will give trend for ones who want to process SVF in areas only covered by the LOD1 BDTOPO database: around $23 \%$ of the SVF values differ of more than 0,02 . Nevertheless, local difference can be very significant in dense urban areas. SVF differences between IDB1 and IDB2 prove that, for such an indicator, roof modeling is important. But, this importance depends on the point altitude. The use SVF with BDTOPO data is relevant if the goal is to have a global average indicator as geometric modeling somehow cancels out roof modeling but, locally, important errors must be considered. In the future, we will explore the possibility to detect automatically buildings that may cause errors in order to find zones in which the indicator value is reliable.

Paradoxically, a LOD1 dataset with footprints from 3DDB will cause more differences than using BDTOPO. Selection influences only locally SVF and only in particular contexts.

Future work will involve testing the method on other indicators (notably built area) and databases (we are looking forward to apply our method on a finer LOD1 and a LOD3 dataset). We will also decompose the accuracy error in planimetric and altimetric errors by moving the walls of BDTopo 
database to match with the footprints of 3DDB. When dealing with the question of isolating sources of uncertainty in the data, several questions arise. In particular, one important question concerns the possibility of using ontology of the sources of uncertainty in order to automatically derive the intermediate databases. Another ontology, of the indicator, could also be used in order to consider the different type of indicators for a more automatic method implementation. SVF considers empty space viewed from a point, some indicators consider only one building (floor built) or relations between buildings (adjacency). In order to automatically compute differences of values for two different datasets, the challenge will be to make the data match and to make relevant comparison.

Another future work is to consider this method in a decision process. We are looking forward to integrate it in a tool for automatic verification of urban regulations [34]. The aim will be to give a confidence measure for the respect of urban regulations rather than only a Boolean value (respected/not respected).

We express our gratitude to the Urban Community of Strasbourg for providing us databases in the context of the ZAEU agreement (Urban Environmental Study Zone).

\section{References}

[1] L. Adolphe, Environment and Planning B: Planning and Design 28(2), 183 (2001)

[2] C. Carneiro, E. Morello, G. Desthieux, F. Golay, Urban environment quality indicators: application to solar radiation and morphological analysis on built area, in Proceedings of the 3rd WSEAS international conference on Visualization, imaging and simulation (World Scientific and Engineering Academy and Society (WSEAS), Stevens Point, Wisconsin, USA, 2010), VIS '10, pp. 141-148, ISBN 978-960-474-246-2

[3] J. Ganitseva, V. Coors, Automatic landmark detection for 3D urban models, in 5th International Conference on 3D GeoInformation (2010)

[4] T.H. Kolbe, G. Gröger, Towards unified 3D city models, in Challenges in Geospatial Analysis, Integration and Visualization II. Proc. of Joint ISPRS Workshop (Stuttgart, 2003)

[5] G.T. Johnson, I.D. Watson, J. Climate Appl. Meteor. 23(2), 329 (1984)

[6] R. Devillers, R. Jeansoulin, Fundamentals of Spatial Data Quality (2006)

[7] W. Kresse, K. Fadaie, ISO standards for geographic information (Springer, 2004)

[8] A. De-La-Losa, Ph.D. thesis (2000)

[9] B. Kazar, R. Kothuri, P. Van Oosterom, S. Ravada, On Valid and Invalid Three-Dimensional Geometries (Springer, 2008), pp. 19-46

[10] R.M. Thompson, V. Oosterom, Modelling and validation of 3D cadastral objects, in 28th urban data management symposium (UDMS 2011) (2011)

[11] J. Bogdahn, V. Coors, Towards and automated healing of 3D urban models, in 5th International Conference on 3D GeoInformation (2010)

[12] V. Coors, M. Krämer, Integrating quality management into a 3D geospatial server, in 28th urban data management symposium (UDMS 2011) (2011)

[13] A. Prévost, D. Rodríguez, N. Molines, B. Beckers, I. Caudron, Optimiser l'efficacité énergétique du bâti et de la ville grâce à la modélisation 3D. Enjeux et perspectives d'application, in Conférence internationale de Géomatique et Analyse Spatiale, SAGEO'10 (2010)

[14] T. Gál, F. Lindberg, J. Unger, 95(1-2), 111 (2009)

[15] M.J. Brown, S. Grimmond, C. Ratti, Comparison of Methodologies for Computing Sky View Factor in Urban Environments (ISEH and IAHR, 2001)

[16] F. Tagliavini, P. Reichenbach, D. Maragna, F. Guzzetti, A. Pasuto, Geoinformatica 13(3), 323 (2009) 
[17] C. Carneiro, E. Morello, T. Voegtle, F. Golay, T. GIS 14(4), 497 (2010)

[18] N.R. Chrisman, B.S. Yandell, Surveying and Mapping 48, 241 (1988)

[19] D.A. Griffith, Distance calculations and errors in geographic databases (Taylor \& Francis, 1989), pp. $81-90$

[20] P. van Oort, Ph.D. thesis, Wageningen University (2005)

[21] J.F. Girres, A. Ruas, Estimation of Imprecision in Length and Area Computation in Vector Databases Including Production Processes Description, in 14th International Symposium on Spatial Data Handling (SDH'10), Joint International Conference on Theory, Data Handling and Modelling in GeoSpatial Information Science (2010)

[22] J.F. Girres, A model to estimate length measurements uncertainty in vector databases, in 7th International Symposium on Spatial 2 Data Quality (2011)

[23] Oke, Energy and Buildings 11(1-3), 103 (1988)

[24] L.C.L. Souza, D.S. Rodrigues, J.F.G. Mendes, Sky View Factors estimation using a 3D-GIS extension, in Eighth International IBPSA Conference (2003)

[25] D. Fisher-Gewirtzman, M. Burt, Y. Tzamir, Environment and Planning B: Planning and Design 30(4), 575 (2003)

[26] J. Unger, 36(1-2-3), 59 (2009)

[27] M.K. Svensson, Meteorological Applications 11(3), 201 (2004)

[28] D. Vettorato, D.C. Prosperi, Specifying spatial attributes and relations in Urban Heat Islands: A generalizable model applied to Trento, Italy, in 28th Urban Data Management Symposium UDMS'11 (Taylor and Francis, 2011), pp. 181-192

[29] J. Teller, Ph.D. thesis, Université de Liège (2001)

[30] F. Sarradin, Ph.D. thesis, Université de Nantes (2004)

[31] E. Morello, C. Ratti, Environment and Planning B: Planning and Design 36(5), 837 (2009)

[32] K. Lynch, The Image of the City (The MIT Press, 1960), ISBN 0262620014

[33] M. Brasebin, GeOxygene: An Open 3D Framework for the Development of Geographic Applications, in 12th International Conference on Geographic Information Science (AGILE) (2009)

[34] M. Brasebin, J. Perret, C. Haëck, Towards a 3D geographic information system for the exploration of urban rules: application to the French local urban planning schemes, in 28th urban data management symposium (UDMS 2011) (2011) 